\title{
L-Dopa gains psychostimulant-like properties after nigral dopaminergic loss
}

\footnotetext{
Michel Engeln 1,2, Stefania Fasano ${ }^{3}$, Serge H. Ahmed ${ }^{1,2}$, Martine Cador ${ }^{4,5}$, Veerle Baekelandt $^{6}$, Erwan Bezard ${ }^{1,2,7, \#}$, Pierre-Olivier Fernagut ${ }^{1,2}$

1 Univ. de Bordeaux, Institut des Maladies Neurodégénératives, UMR 5293, F-33000 Bordeaux, France; ${ }^{2}$ CNRS, Institut des Maladies Neurodégénératives, UMR 5293, F-33000 Bordeaux, France; ${ }^{3}$ Institute of Experimental Neurology, Division of Neuroscience, San Raffaele Research Institute, 20132 Milano, Italy; 4 Univ. de Bordeaux, Institut de Neurosciences Cognitives et Intégratives d'Aquitaine, UMR5287, F-33000 Bordeaux, France; ${ }^{5}$ CNRS, Institut de Neurosciences Cognitives et Intégratives d'Aquitaine, UMR5287, F-33000 Bordeaux, France; ${ }^{6}$ Laboratory for Neurobiology and Gene Therapy, Katolik University, B-3000 Leuven, Belgium ; ${ }^{7}$ Service de Neurologie, CHU de Bordeaux, F33604 Pessac, France.
}

\# : Corresponding author : Erwan Bezard, CNRS UMR 5293, Institute of Neurodegenerative Diseases, Université Bordeaux Segalen, 146 rue Léo Saignat, 33076 Bordeaux cedex, France, Tel: +33 557571 685, Fax: +33 556986 182; E-mail: erwan.bezard@u-bordeaux2.fr

\section{Running head: Psychostimulant-like properties of L-Dopa}

Number of characters in the title: 75

Number of characters in the running head: 41

Number of words in the abstract: 99

Number of words in the body of the manuscript: 1500

Number of figures: 3

Number of tables: 0

This article has been accepted for publication and undergone full peer review but has not been through the copyediting, typesetting, pagination and proofreading process which may lead to differences between this version and the Version of Record. Please cite this article as an 'Accepted Article', doi: 10.1002/ana.23881 


\section{Abstract}

Dopamine Dysregulation Syndrome shares some core behavioral features of psychostimulant addiction, suggesting that dopamine replacement therapy can acquire psychostimulant-like properties in some patients with Parkinson's disease (PD). We here report strong experimental evidence supporting this hypothesis in an alpha-synuclein rat model of PD. Though L-Dopa had no effect in controls, it acquired two prominent psychostimulant-like properties in Parkinsonian rats: i) it produced intense reward on its own and in parallel ii) decreased interest in other nondrug reward. These two effects may combine to explain the addictive use of L-Dopa after loss of midbrain dopamine neurons in some PD patients.

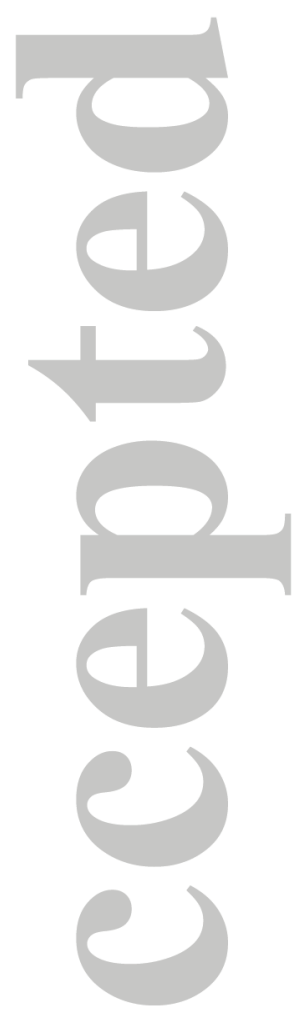

Keywords: Alpha-synuclein, Dopamine Dysregulation Syndrome, Reward, Saccharin, Conditioned Place Preference 


\section{INTRODUCTION}

Symptomatic treatment of Parkinson's disease (PD) with dopamine replacement therapy (DRT) is not without important limits. Besides its inability for alleviating non-motor symptoms, chronic DRT leads to a number of motor (e.g. dyskinesia and motor fluctuations) and nonmotor side-effects. Non-motor side-effects include the dopamine dysregulation syndrome (DDS) occurring in $3-4 \%$ of PD patients taking levodopa and/or apomorphine. DDS is characterized by compulsive DRT-seeking and hoarding, self-medication and withdrawal syndrome ${ }^{1}$. Growing evidences for an activation of the reward pathways by different types of DRT have been provided ${ }^{2}$. Compulsive DRT use shares features with psychostimulant addiction ${ }^{3}$. This study therefore investigated whether L-Dopa possesses psychostimulantlike properties by evaluating its rewarding properties using conditioned place preference (CPP) and its ability to affect a nondrug reward in a sweet-taste preference paradigm. The influence of an altered dopaminergic function on the emergence of compulsive DRT use ${ }^{4}$ was assessed using bilateral lesions of the Substantia Nigra pars compacta (SNc) induced by viral-mediated overexpression of alpha-synuclein.

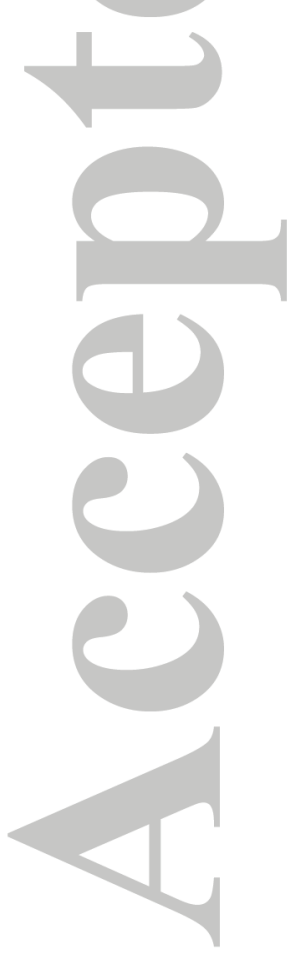




\section{MATERIALS AND METHODS}

\section{Animals}

Fifty-three male Wistar Han rats (Charles River Laboratories, France) with water and regular rodent chow available ad libitum were used. All experiments were performed in accordance with French (87-848, Ministère de l'Agriculture et de la Forêt) and European Communities Council Directive (2010/63/EU) guidelines for the care of laboratory animals and were approved by the institutional Ethical Committee.

\section{Stereotaxic viral-mediated lesion}

Experimental parkinsonism was achieved with a bilateral lesion of the SNc using an AAV2-9 vector expressing human A53T a-synuclein under the control of synapsin I promoter (7.0 $\mathrm{x} 10^{12} \mathrm{vg} / \mathrm{ml}$ ). Sham rats received an AAV2-9 vector expressing the green fluorescent protein (GFP; $2.85 \times 10^{12} \mathrm{vg} / \mathrm{ml}$ ). Motor function was assessed using the stepping test ${ }^{5}$ at baseline, 8 weeks after surgery, and at the end of the third CPP pairing with L-Dopa. Alpha-synuclein overexpression in parkinsonian rats induced a significant bilateral stepping deficit $(-24.05 \%$; Wilcoxon; $p<0.001$ from Vehicle) significantly improved with $12 \mathrm{mg} / \mathrm{kg}$ L-Dopa i.p. $(+15.4 \%$; Wilcoxon; $p<0.01$ from Vehicle) (Fig 1A).

Rats were euthanized with a lethal injection of chloral hydrate $(600 \mathrm{mg} / \mathrm{kg}, \mathrm{VWR}$, France) and perfused with $4 \%$ paraformaldehyde (PFA). $40 \mu$ m-thick free-floating serial coronal sections were collected throughout the mesencephalon and processed for tyrosine hydroxylase (TH), Ubiquitin and Synuclein immunohistochemistry with nissl counterstaining. Unbiased stereological sampling was used to estimate the total number of $\mathrm{TH}$-positive neurons and Nissl-stained cells in the SNc and VTA using Mercator v7.8.9 (Explora Nova, France) ${ }^{6}$. Viralmediated expression of alpha-synuclein induced a $30.7 \%$ loss of dopaminergic neurons in the SNc (t-test; $t=5.887, d f=51 ; p<0.0001)($ Fig 1B-C). 


\section{Conditioned Place Preference for L-Dopa}

CPP apparatus consisted in two square chambers $(30 \times 30 \times 45 \mathrm{~cm})$ linked by a central neutral room $(30 \times 15 \times 45 \mathrm{~cm})$ with two 'guillotine' doors. Each chamber had a unique configuration with different visual patterns on the walls and a different floor texture. The apparatus was homogeneously enlightened with dim light (30 lux; protocol adapted from ${ }^{7}$ ). For the conditioning, 35 animals received either L-Dopa $(12 \mathrm{mg} / \mathrm{kg}+$ a DOPA decarboxylase inhibitor: benserazide $15 \mathrm{mg} / \mathrm{kg}$ i.p. in $0.9 \%$ saline; both from Sequoia Research Products, UK) or benserazide alone (protocol detailed in the legend to Fig 2). Place preference scores were calculated as the difference in time spent in the drug-paired chamber between probe test and pretest. Rats that spent more than $70 \%$ of the time in the central compartment during probe test were excluded.

\section{Sweet taste preference}

Sweet taste preference ${ }^{8}$ was performed in single cages $(50 \times 25 \times 20 \mathrm{~cm})$ equipped with two drinking spouts containing $0.5 \mathrm{~mL}$ of either tap or saccharin (Sigma-Aldrich, France) sweetened-water with automatic refilling (Imetronic, France). Schedule of the experiment: training of the 18 rats for 9 days $(0.2 \%$ saccharin), surgery ( +8 weeks for alpha-synuclein accumulation), 9 days of rebaselining ( $0.2 \%$ saccharin), doses-response curve $(0.04 \%$, $0.008 \%$ and $0.0016 \%$ saccharin; alternated with $0.2 \%$ saccharin) and drug test (L-Dopa: 0,6 and $12 \mathrm{mg} / \mathrm{kg}$, injected i.p $30 \mathrm{~min}$ before the test; alternated with vehicle sessions).

\section{Data analysis}

Data were subjected to a Wilcoxon matched pairs test for stepping test. Stereological counts and CPP scores were analyzed using Student unpaired t-test. For sweet-taste preference data, two-way ANOVA and two-way ANOVA with repeated-measure (RM-ANOVA) were run with Newman-Keuls post-hoc test. All data are presented as mean \pm SEM with a threshold for statistical significance at $p<0.05$. 


\section{RESULTS}

\section{Rewarding properties of L-Dopa in lesioned animals}

While L-Dopa did not induce a place preference in sham animals (Fig 2A), it induced a significant place preference in bilaterally lesioned rats $(t=2.17, d f=15 ; p<0.05)$ (Fig 2B), indicating that L-Dopa is rewarding only in parkinsonian animals.

\section{L-Dopa decreases sweet taste preference in lesioned animals}

During baseline Saccharin $(0.2 \%)$ discrimination training, consumption rapidly increased as indicated by RM-ANOVA (Session: $F_{[8,161]}=11.89 ; p<0.001$ ). From session 2 onwards, saccharin intake significantly differed from first session in both groups (Newman-Keuls; p<0.05) (Fig 3A). After the lesion, saccharin (0.2\%) consumption was re-baselined (Fig 3A). No differences between groups and no differences between all re-baselining sessions and the last training session were found, indicating that the lesion had no effect on the discrimination capacities of the rats at this dose (Fig $\mathbf{3 A}$ ). We then tested the preservation of discriminative abilities after the lesion (Fig 3A). Two-way ANOVA reported a concentration effect $\left(F_{[3,71]}=41.64 ; p<0.001\right)$ but no difference between groups for the 3 different doses $(0.04 \% ; 0.008 \%$ and $0.0016 \%)$. Animals from both groups drunk significantly less $0.04 \%$ saccharin compared with $0.2 \%$ but significantly more compared with $0.008 \%$ and $0.0016 \%$ (Newman-Keuls; $p<0.05$ for each, Fig 3A). The $0.04 \%$ concentration was thus chosen to avoid a ceiling effect.

Different L-Dopa doses were then tested on saccharin intake. Two-way ANOVA revealed a significant effect of lesion $\left(F_{[1,53]}=8.35 ; p<0.01\right)$ and post-hoc analysis highlighted a significant difference between both groups for the $12 \mathrm{mg} / \mathrm{kg}$ dose (Newman-Keuls; $p<0.05$, Fig 3B). Differential saccharin intake during L-Dopa sessions highlighted a general decrease in saccharin consumption for lesioned rats whereas sham animals remained at comparable level regardless of the L-Dopa dose (one sample $t$-test: $t=2.82, d f=9 ; p<0.05$; Fig $3 \mathbf{C}$ ). Correction for multiple comparisons would however reach only trend level $(p=0.07)$, suggesting the finding requires further replication. In cumulated saccharin consumption 
following $12 \mathrm{mg} / \mathrm{kg}$ L-Dopa, an interaction between Group and Time $\left(F_{[9,179]}=3.87 ; p<0.001\right)$ indicates that bilaterally lesioned animals drank significantly less saccharin from minute 18 onwards compared with sham (Newman-Keuls; $p<0.05)$. No significant increase from minute 18 to minute 27 (Fig 3D) indicates that L-Dopa-treated bilaterally lesioned animals displayed a reduced interest for a natural reward.

\section{DISCUSSION}

The present study demonstrates rewarding properties of L-Dopa at therapeutically relevant doses. Interestingly, such rewarding effect was observed only in lesioned animals, indicating that midbrain neurodegeneration is critical for the expression of this behavior. Moreover, LDopa decreased sweetened-water consumption in lesioned rats suggesting that, like psychostimulants, L-Dopa affects the palatability of a nondrug reward.

In patients suffering from DDS, L-Dopa is reported to share behavioral and affective features of cocaine or amphetamine addiction and induce similar withdrawal signs ${ }^{1-3} 9$. Interestingly in healthy subjects, L-Dopa fails to generate a psychostimulant-like mood elevation ${ }^{10}$. To test whether dopaminergic loss may reveal psychostimulant-like properties of L-Dopa, we used a bilateral lesion model induced by viral-mediated accumulation of alpha-synuclein in SNc with L-Dopa-responsive motor impairments. Our CPP experiment revealed that a L-Dopa dose that alleviates motor symptoms was rewarding exclusively in lesioned rats. Such differential effect might reflect a supersensitivity of post-synaptic receptors ${ }^{11-13}$ accounting for a preferential action of L-Dopa on altered dopaminergic pathways as occurring in PD ${ }^{1,14}$.

An optimal dopaminergic activity is required for expressing both motivation and rewarding properties of food ${ }^{15,16}$. In our experiments, the dopaminergic lesion alone did affect neither discrimination between tap and sweetened-water nor the palatability of saccharin. However, after L-Dopa administration, while preference for saccharin was preserved, the lesioned animals drank significantly less sweetened-water than shams. Previous work by Heffner et al. ${ }^{15}$ showed that L-Dopa or amphetamine in 6-hydroxydopamine lesioned rats had the same anorexic effect as amphetamine in normal rat. Here, we can extend these observations to a 
non-caloric reward, accounting for mechanisms linked to motivation and/or hedonic perception rather than to satiety. L-Dopa might have 'overdosed' the altered nigrostriatal pathway leading to a diminished interest for saccharin. As previously proposed ${ }^{15,17}$, drugs that overflow the dopaminergic activity might outweigh the prominence of gustatory reward leading to a decrease in their consumption. Whether mechanisms involved in psychostimulant-induced devaluation of natural rewards similarly operate with L-Dopa will need to be addressed in future studies.

Together, these data demonstrate that, at a therapeutic dose, L-Dopa has rewarding properties and can reduce the consumption of a nondrug reward in individuals with a dopaminergic lesion restricted to the SNc. These psychostimulant behavioral features suggest that dopaminergic neurodegeneration triggers the emergence of "cocaine-like" properties of L-Dopa, which may contribute to the subsequent development of DDS. Whether additional degeneration of VTA neurons can trigger DDS by enhancing such psychostimulant-like properties in vulnerable individuals needs to be assessed. Experimental models of PD are thus amenable to investigate the cellular and molecular mechanisms leading to compulsive use of L-Dopa as well as to identify predictive and inductive factors that may lead to DDS.

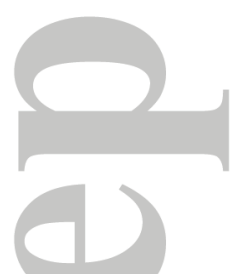

\section{ACKNOWLEDGEMENTS}

This work was supported by Agence Nationale de la Recherche grants (EB: ANR-07-MNPTrafinlid). We thank Stéphane Lelgouach, Sandra Dovero and Alain Estager for their excellent technical support and Ourida Gaucher for administrative support. The Université Bordeaux Segalen and the Centre National de la Recherche Scientifique provided infrastructural support. 


\section{FIGURE LEGENDS}

Figure 1: Viral-mediated lesion of Substantia Nigra: AAV2-9 vector expressing human A53T a-synuclein and AAV2-9 vector expressing the green fluorescent protein (GFP; for sham rats) were injected under isoflurane anesthesia in two rostrocaudal sites in each $\mathrm{SNc}(1 \mu \mathrm{L}$ per site) at the following coordinates (in mm from bregma and dura) ${ }^{18}$ : $\mathrm{AP}-4.9, \mathrm{ML}+/-2.2$, DV -8 and AP -5.4, ML +/-2, DV -8. (A) Motor impairments measured by stepping test before the surgery (white), after the surgery (dark gray; Wilcoxon ${ }^{*} p<0.05$ from before surgery), with benserazide injection ('OFF-state': light gray; * $p<0.05$ from before the surgery) and after the surgery with L-Dopa 12mg/kg injection ('ON-state': middle-shaded gray; $\# p<0.01$ from after the surgery with benserazide injection) (Mean \pm SEM; triangles represent 'Vehicle' animals and dots represent 'L-Dopa-treated' animals); (B) Stereological counts of TH positive cells in Substantia Nigra pars compacta (SNc) and Ventral Tegmental Area (VTA) $\left({ }^{*} p<0.0001\right.$ from sham) (Mean \pm SEM) ; (C) Histological characterization of the viral-mediated alpha-synuclein overexpression highlighted: a loss of TH-positive neurons in both striatum and $\mathrm{SNc}\left(1^{\text {st }}\right.$ column: striatum: pseudo-color; monoclonal mouse anti-TH antibody MAB 318 Milipore; $2^{\text {nd }}$ column: SNc: magnification X2.5; monoclonal mouse anti-TH antibody MAB 318 Milipore, France; $3^{\text {rd }}$ column: SNc: magnification X40; polyclonal sheep anti-TH antibody Ab113 Abcam, UK + polyclonal donkey anti-goat alexa 488 Invitrogen, USA), ubiquitin inclusion in TH-positive SNc neurons of lesioned rats $\left(4^{\text {th }}\right.$ column: magnification X40; polyclonal rabbit anti-Ubiquitin U5379 Sigma-Aldrich, France + anti-rabbit Envision system-HRP labeled polymer DAKO, USA + anti-HRP Dylight 549 Jackson ImmunoResearch, USA), synuclein accumulation in remaining $\mathrm{TH}$-positive SNc cells of lesioned animals $\left(5^{\text {th }}\right.$ column: magnification X40; monoclonal mouse anti-synuclein Neo marker MS-1572-P1 Thermo Scientific,UK + polyclonal goat anti-mouse Alexa Cys5 Invitrogen, USA), merge (6 $6^{\text {th }}$ column). Our viral-mediate lesion model exhibits some of the cellular and behavioral features present in PD. Thus, in addition to induce TH-positive cell death in SNc, alpha-synuclein accumulation certainly participated to worsen stepping test performances by causing 
inflammation, proteasome and lysosomal impairments and synaptic dysfunction leading to axonopathy in the surviving neurons as shown by ${ }^{19,20}$.

Figure 2: Rewarding properties of L-Dopa in lesioned rats: The first day, a 20min habituation period allowed exploration of the apparatus (pretest). Time spent in each chamber was analyzed using EthoVision v3 (Noldus Information Technology, the Netherlands). Time spent in each room during pretest was used to determine the drug-paired chamber. The following days, animals received either L-Dopa on days 1,3,5, and 7 or Vehicle (benserazide) on days 2, 4, 6 and 8 and were placed in the paired-chamber for 40 minutes. Controls groups received benserazide during all conditioning sessions. The day following the last conditioning session, a probe test was performed with $20 \mathrm{~min}$ free access to the apparatus. CPP scores were calculated for each animal as follow: (time in the drug side $\mathrm{X}$ session time) / (time in left side + time in right side). (A) CPP score in sham animals for Vehicle and L-Dopa $(12 \mathrm{mg} / \mathrm{kg}$; n=10 and 8 respectively) ; (B) CPP score in lesioned animals for Vehicle and L-Dopa $\left(12 \mathrm{mg} / \mathrm{kg} ; \mathrm{n}=8\right.$ and 9 respectively) $\left({ }^{*} \mathrm{p}<0.05\right)$ (Mean \pm SEM for both graphs).

Figure 3: L-Dopa decreases sweet taste preference: In the test cages, position of sweetened-water was counter-balanced every day. Rats acquired the task during 9 daily 30 min sessions, choosing between tap and $0.2 \%$ saccharin sweetened-water. 8 weeks after surgery (dashed line illustrates the surgery), rats were re-baselined for 9 additional days. Dose-response curve was established on following days using $0.04 \%, 0.008 \%$ and $0.0016 \%$ saccharin alternated with $0.2 \%$ saccharin sessions. Finally, doses of L-Dopa $(0,6$ and $12 \mathrm{mg} / \mathrm{kg}$, injected $30 \mathrm{~min}$ before the test) alternated with vehicle sessions. (A) Saccharin consumption during training, re-baselining (saccharin $0.2 \%$; $n=8$ for sham and $n=10$ for lesioned animals) (\#p<0.05 from session 1 for both groups) and dose response test; (B) Effect of L-Dopa on saccharin $(0.04 \%)$ intake $\left({ }^{*} \mathrm{p}<0.05\right.$ from sham) ; (C) Differential saccharin intake (i.e. intake for $6 \mathrm{mg} / \mathrm{kg}$ - intake for $0 \mathrm{mg} / \mathrm{kg} \mathrm{L-Dopa} \mathrm{and} \mathrm{intake} \mathrm{for} 12 \mathrm{mg} / \mathrm{kg}$ - intake for Omg/kg L-Dopa): positive score indicates an increased intake while a negative score indicate 
a decreased intake. 0 indicates no changes between both sessions. Colors represent individual animals and lines represent mean \pm SEM $(\$ p<0.05$ from 0$)$. (D) Cumulated saccharin $(0.04 \%)$ consumption for the $12 \mathrm{mg} / \mathrm{kg} \mathrm{L-Dopa} \mathrm{session}\left({ }^{*} p<0.05\right.$ from sham).

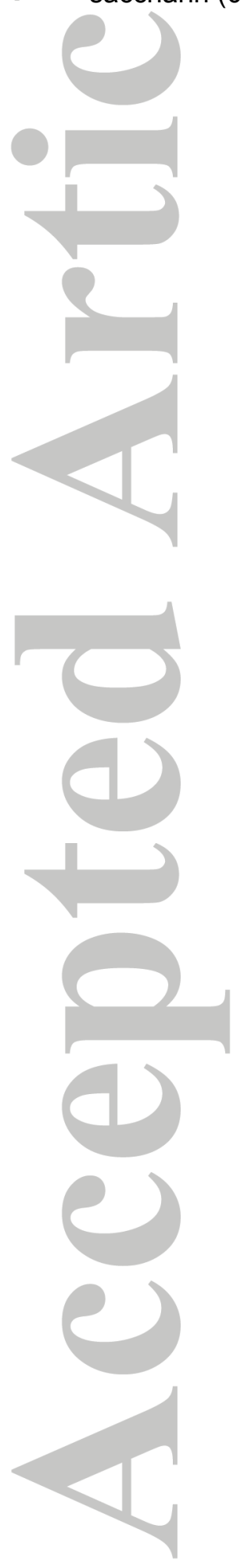




\section{REFERENCES}

1. Lawrence $A D$, Evans $A H$, Lees AJ. Compulsive use of dopamine replacement therapy in Parkinson's disease: reward systems gone awry? Lancet Neurol. 2003 Oct;2(10):595-604.

2. O'Sullivan SS, Evans AH, Lees AJ. Dopamine dysregulation syndrome: an overview of its epidemiology, mechanisms and management. CNS Drugs. 2009;23(2):157-70.

3. Giovannoni G, O'Sullivan JD, Turner K, Manson AJ, Lees AJ. Hedonistic homeostatic dysregulation in patients with Parkinson's disease on dopamine replacement therapies. J Neurol Neurosurg Psychiatry. 2000 Apr;68(4):423-8.

4. Voon V, Fernagut PO, Wickens J, et al. Chronic dopaminergic stimulation in Parkinson's disease: from dyskinesias to impulse control disorders. Lancet Neurol. 2009 Dec;8(12):1140-9.

5. Olsson M, Nikkhah G, Bentlage C, Bjorklund A. Forelimb akinesia in the rat Parkinson model: differential effects of dopamine agonists and nigral transplants as assessed by a new stepping test. J Neurosci. 1995 May;15(5 Pt 2):3863-75.

6. Engeln M, Ahmed SH, Vouillac C, Tison F, Bezard E, Fernagut PO. Reinforcing properties of Pramipexole in normal and parkinsonian rats. Neurobiol Dis. 2012 Aug 23;49C:79-86.

$7 . \quad$ Fasano S, D'Antoni A, Orban PC, et al. Ras-guanine nucleotide-releasing factor 1 (Ras-GRF1) controls activation of extracellular signal-regulated kinase (ERK) signaling in the striatum and longterm behavioral responses to cocaine. Biol Psychiatry. 2009 Oct 15;66(8):758-68.

8. Vendruscolo LF, Gueye AB, Darnaudery M, Ahmed SH, Cador M. Sugar overconsumption during adolescence selectively alters motivation and reward function in adult rats. PLoS One. 2010;5(2):e9296.

9. Evans AH, Lawrence AD, Cresswell SA, Katzenschlager R, Lees AJ. Compulsive use of dopaminergic drug therapy in Parkinson's disease: reward and anti-reward. Mov Disord. 2010 May 15;25(7):867-76.

10. Liggins J, Pihl RO, Benkelfat C, Leyton M. The dopamine augmenter L-DOPA does not affect positive mood in healthy human volunteers. PLoS One. 2012;7(1):e28370.

11. Iversen SD, Creese I. Behavioral correlates of dopaminergic supersensitivity. Advances in neurology. 1975;9:81-92.

12. Costall B, Naylor RJ. Actions of dopaminergic agonists on motor function. Advances in neurology. 1975;9:285-97.

13. Roberts DC, Koob GF, Klonoff P, Fibiger HC. Extinction and recovery of cocaine selfadministration following 6-hydroxydopamine lesions of the nucleus accumbens. Pharmacol Biochem Behav. 1980 May;12(5):781-7.

14. Evans $A H$, Pavese $N$, Lawrence $A D$, et al. Compulsive drug use linked to sensitized ventral striatal dopamine transmission. Ann Neurol. 2006 May;59(5):852-8.

15. Heffner TG, Zigmond MJ, Stricker EM. Effects of dopaminergic agonists and antagonists of feeding in intact and 6-hydroxydopamine-treated rats. J Pharmacol Exp Ther. 1977 May;201(2):38699.

16. Wise RA. Dual Roles of Dopamine in Food and Drug Seeking: The Drive-Reward Paradox. Biol Psychiatry. 2012 Oct 5.

17. Volkow ND, Fowler JS, Wang GJ, Swanson JM. Dopamine in drug abuse and addiction: results from imaging studies and treatment implications. Mol Psychiatry. 2004 Jun;9(6):557-69.

18. Paxinos G, Watson C. The rat brain in stereotaxic coordinates. 6th ed: Elsevier; 2009.

19. Lundblad M, Decressac M, Mattsson B, Bjorklund A. Impaired neurotransmission caused by overexpression of alpha-synuclein in nigral dopamine neurons. Proc Natl Acad Sci U S A. 2012 Feb 28;109(9):3213-9.

20. Decressac M, Mattsson B, Bjorklund A. Comparison of the behavioural and histological characteristics of the 6-OHDA and alpha-synuclein rat models of Parkinson's disease. Exp Neurol. 2012 May;235(1):306-15. 
A

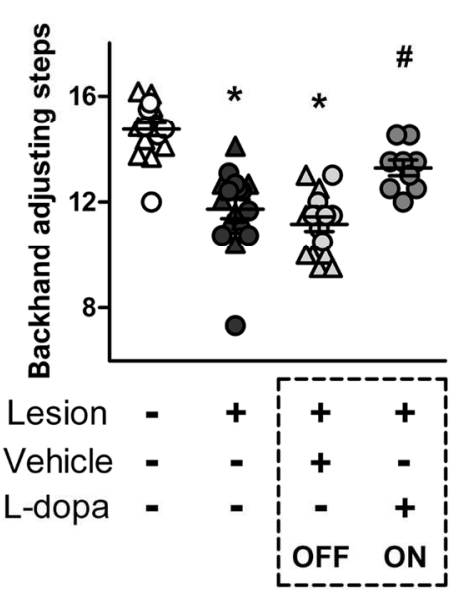

B

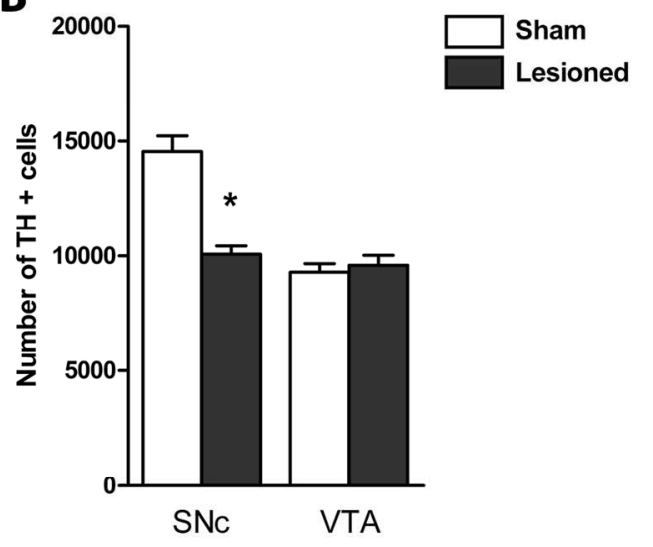

C TH low power

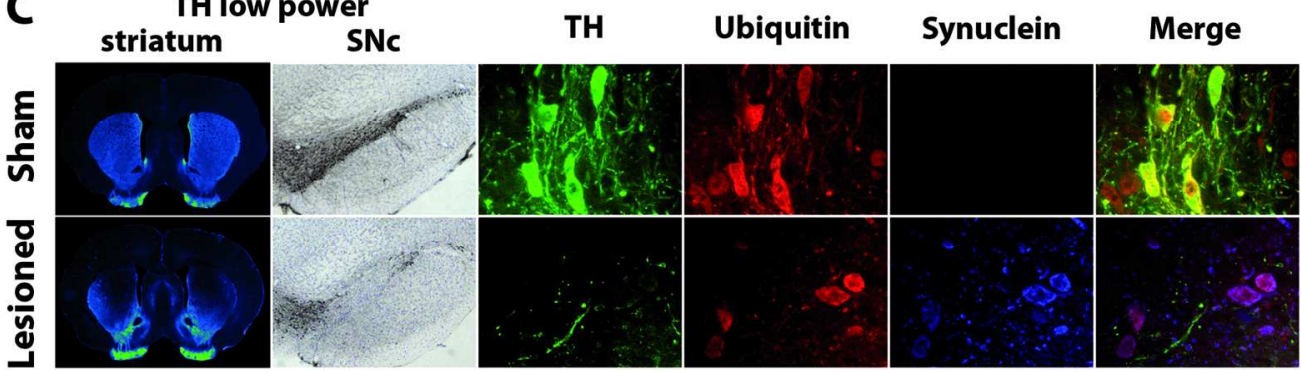

Figure 1: Viral-mediated lesion of Substantia Nigra: AAV2-9 vector expressing human A53T a-synuclein and AAV2-9 vector expressing the green fluorescent protein (GFP; for sham rats) were injected under isoflurane anesthesia in two rostrocaudal sites in each SNc (1 $\mu \mathrm{L}$ per site) at the following coordinates (in $\mathrm{mm}$ from bregma and dura) 18: AP -4.9, ML +/-2.2, DV -8 and AP -5.4, ML +/-2, DV -8. (A) Motor impairments measured by stepping test before the surgery (white), after the surgery (dark gray; Wilcoxon $* \mathrm{p}<0.05$ from before surgery), with benserazide injection ('OFF-state': light gray; ${ }^{*} p<0.05$ from before the surgery) and after the surgery with L-Dopa $12 \mathrm{mg} / \mathrm{kg}$ injection ('ON-state': middle-shaded gray; $\# \mathrm{p}<0.01$ from after the surgery with benserazide injection) (Mean \pm SEM; triangles represent 'Vehicle' animals and dots represent 'L-Dopa-treated' animals); (B) Stereological counts of TH positive cells in Substantia Nigra pars compacta

$(\mathrm{SNc})$ and Ventral Tegmental Area (VTA) $(* \mathrm{p}<0.0001$ from sham) (Mean \pm SEM) ; (C) Histological characterization of the viral-mediated alpha-synuclein overexpression highlighted: a loss of TH-positive neurons in both striatum and SNc (1st column: striatum: pseudo-color; monoclonal mouse anti-TH antibody MAB 318 Milipore; 2nd column: SNc: magnification X2.5; monoclonalmouse anti-TH antibody MAB 318 Milipore, France; 3rd column: SNc: magnification X40; polyclonal sheep anti-TH antibody Ab113 Abcam, UK + polyclonal donkey anti-goat alexa 488 Invitrogen, USA), ubiquitin inclusion in TH-positive SNc neurons of lesioned rats (4th column: magnification X40; polyclonal rabbit anti-Ubiquitin U5379 Sigma-Aldrich, France + anti-rabbit Envision system-HRP labeled polymer DAKO, USA + anti-HRP Dylight 549 Jackson ImmunoResearch, USA), synuclein accumulation in remaining TH-positive SNc cells of lesioned animals (5th column: magnification X40; monoclonal mouse anti-synuclein Neo marker MS-1572-P1 Thermo Scientific, UK + polyclonal goat anti-mouse Alexa Cys5 Invitrogen, USA), merge (6th column).

Our viral-mediate lesion model exhibits some of the cellular and behavioral features present in PD. Thus, in addition to induce $\mathrm{TH}$-positive cell death in SNc, alpha-synuclein accumulation certainly participated to worsen stepping test performances by causing inflammation, proteasome and lysosomal impairments and synaptic dysfunction leading to axonopathy in the surviving neurons as shown by 19, 20.

$170 \times 129 \mathrm{~mm}(300 \times 300 \mathrm{DPI})$ 

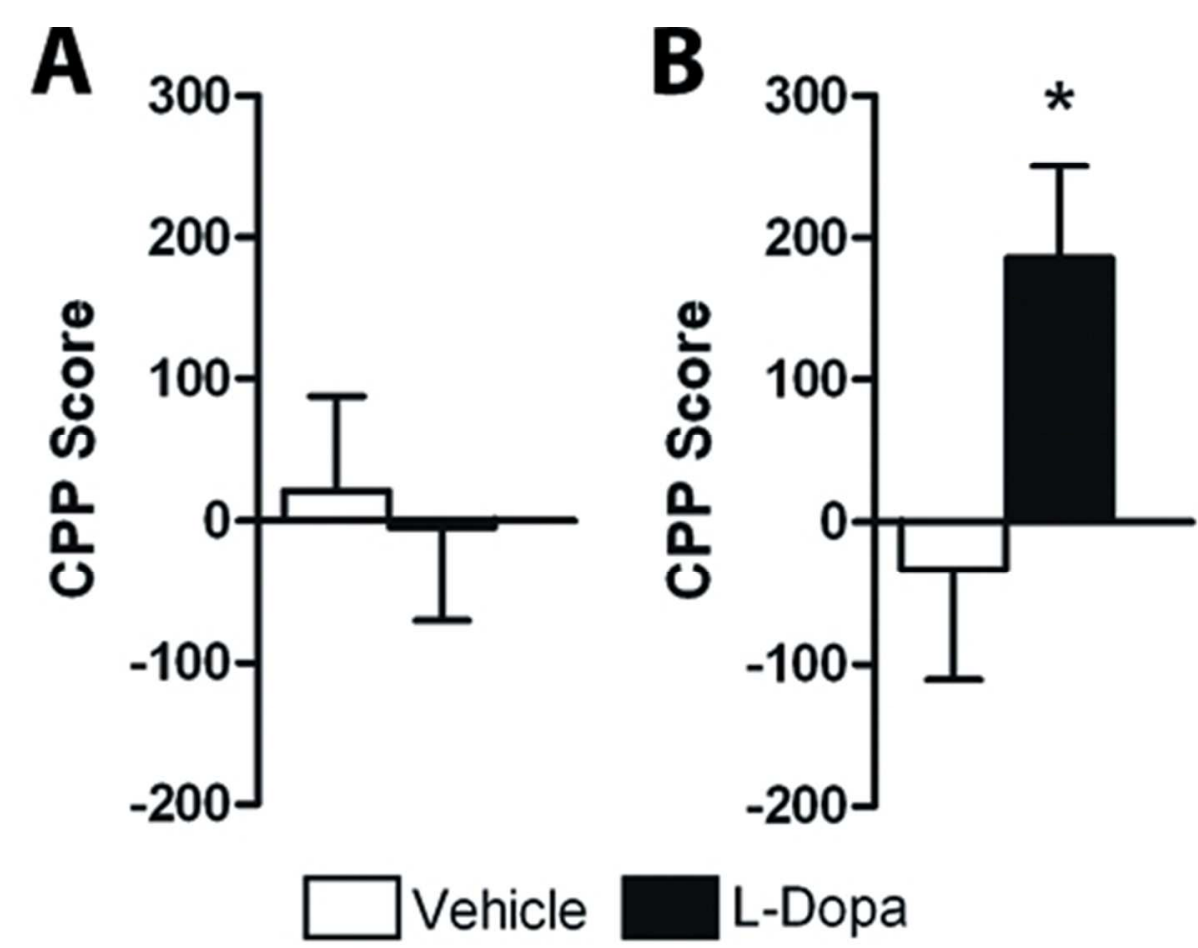

Rewarding properties of L-Dopa in lesioned rats: The first day, a 20min habituation period allowed exploration of the apparatus (pretest). Time spent in each chamber was analyzed using EthoVision v3 (Noldus Information Technology, the Netherlands). Time spent in each room during pretest was used to determine the drug-paired chamber. The following days, animals received either L-Dopa on days $1,3,5$, and 7 or Vehicle (benserazide) on days 2, 4, 6 and 8 and were placed in the paired-chamber for 40 minutes. Controls groups received benserazide during all conditioning sessions. The day following the last conditioning session, a probe test was performed with $20 \mathrm{~min}$ free access to the apparatus. CPP scores were calculated for each animal as follow: (time in the drug side $X$ session time) / (time in left side + time in right side). (A) CPP score in sham animals for Vehicle and L-Dopa $(12 \mathrm{mg} / \mathrm{kg} ; \mathrm{n}=10$ and 8 respectively) ; (B) CPP score in lesioned animals for Vehicle and L-Dopa $(12 \mathrm{mg} / \mathrm{kg} ; \mathrm{n}=8$ and 9 respectively) $(* p<0.05)$ (Mean \pm SEM for both graphs).

$79 \times 61 \mathrm{~mm}(150 \times 150 \mathrm{DPI})$

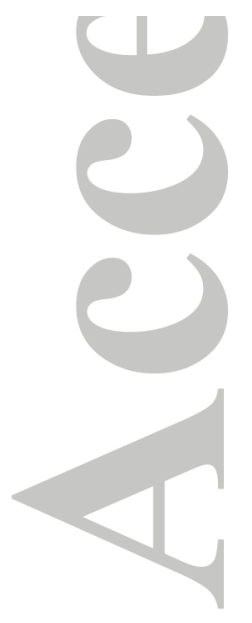


A
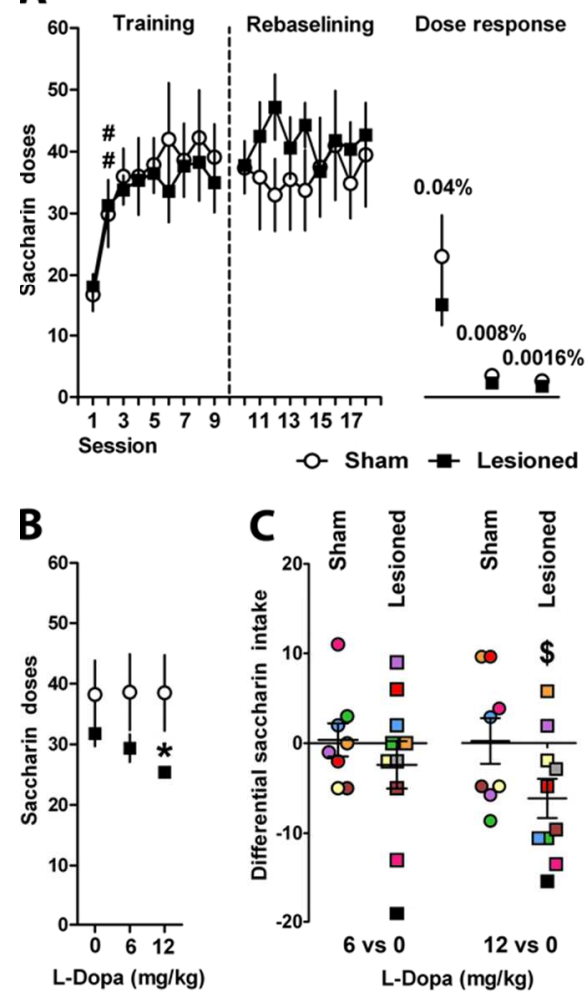

D

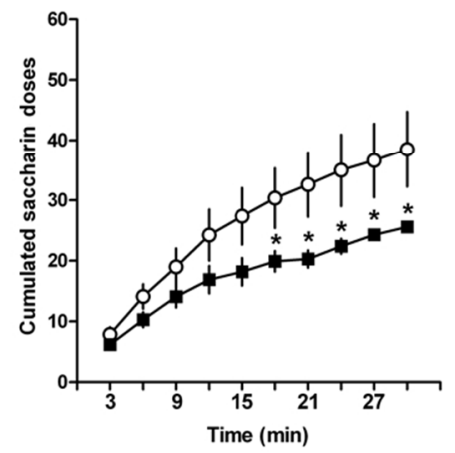

L-Dopa decreases sweet taste preference: In the test cages, position of sweetened-water was counterbalanced every day. Rats acquired the task during 9 daily 30min sessions, choosing between tap and $0.2 \%$ saccharin sweetened-water. 8 weeks after surgery (dashed line illustrates the surgery), rats were rebaselined for 9 additional days. Dose-response curve was established on following days using $0.04 \%$, $0.008 \%$ and $0.0016 \%$ saccharin alternated with $0.2 \%$ saccharin sessions. Finally, doses of L-Dopa (0, 6 and $12 \mathrm{mg} / \mathrm{kg}$, injected $30 \mathrm{~min}$ before the test) alternated with vehicle sessions. (A) Saccharin consumption during training, re-baselining (saccharin $0.2 \% ; n=8$ for sham and $n=10$ for lesioned animals) $(\# p<0.05$ from session 1 for both groups) and dose response test; (B) Effect of L-Dopa on saccharin (0.04\%) intake $<\left({ }^{*} \mathrm{p}<0.05\right.$ from sham) ; (C) Differential saccharin intake (i.e. intake for $6 \mathrm{mg} / \mathrm{kg}$ - intake for $0 \mathrm{mg} / \mathrm{kg}$ L-Dopa and intake for $12 \mathrm{mg} / \mathrm{kg}$ - intake for $0 \mathrm{mg} / \mathrm{kg} \mathrm{L-Dopa):} \mathrm{positive} \mathrm{score} \mathrm{indicates} \mathrm{an} \mathrm{increased} \mathrm{intake} \mathrm{while} \mathrm{a}$ negative score indicate a decreased intake. 0 indicates no changes between both sessions. Colors represent individual animals and lines represent mean \pm SEM $(\$ p<0.05$ from 0$)$. (D) Cumulated saccharin $(0.04 \%)$ consumption for the $12 \mathrm{mg} / \mathrm{kg}$ L-Dopa session $(* \mathrm{p}<0.05$ from sham). 